\title{
A fractional study of generalized Oldroyd-B fluid with ramped conditions via local \& non-local kernels
}

https://doi.org/10.1515/nleng-2021-0013

Received Feb 19, 2021; accepted Jun 9, 2021.

Abstract: Convective flow is a self-sustained flow with the effect of the temperature gradient. The density is nonuniform due to the variation of temperature. The effect of the magnetic flux plays a major role in convective flow. The process of heat transfer is accompanied by mass transfer process; for instance condensation, evaporation and chemical process. Due to the applications of the heat and mass transfer combined effects in different field, the main aim of this paper is to do comprehensive analysis of heat and mass transfer of MHD unsteady Oldroyd-B fluid in the presence of ramped conditions. The new governing equations of MHD Oldroyd-B fluid have been fractionalized by means of singular and non-singular differentiable operators. In order to have an accurate physical significance of imposed conditions on the geometry of Oldroyd-B fluid, the ramped temperature, concentration and velocity are considered. The fractional solutions of temperature, concentration and velocity have been investigated by means of integral transform and inversion algorithm. The influence of physical parameters and flow is analyzed graphically via computational software (MATHCAD-15). The velocity profile decreases by increasing the Prandtl number. The existence of a Prandtl number may reflect the control of the thickness and enlargement of the thermal effect. The classical calculus is assumed as the instant rate of change of the output when the input level changes. Therefore it is not able to include the previous state of the system called the memory effect. Due to this reason, we applied the modern definition of fractional derivatives. Obtained generalized results are very important due to their vast applications in the field of engineering and applied sciences.

Keywords: Oldroyd-B fluid, ramped velocity, porous medium, Laplace transformation, local \& non-local kernels

\footnotetext{
*Corresponding Author: Syed Tauseef Saeed, Department of Science \& Humanities, National University of Computer and Emerging Sciences, Lahore Campus, 54000, Pakistan,
}

\section{Introduction}

Convective flow is a self-sustained flow that transfers heat energy into or out of the body by actual movement of fluids particles that move energy with its mass. Thermal radiation and the effect of magnetic flux plays an important role in convective flow. The different industrial problems and fluid flow in the porous medium have achieved consideration in recent years. In the literature, different theories are made to see the phenomenon of heat transfer analysis. The convection heat transform between two heated cubes discusses by Mousazadeh et al. [1]. Sajad et al. [2] investigate the heat transfer and magnetic effect on hybrid nanofluids. Nazish et al. [3] analyze the influence of heat and mass transform with a magnetic field in the rate type fluid model. The analysis of heat transfer mathematical model's subject to the slip boundary condition for the Maxwell fluid discussed by Han et al. [4]. They explored the exact solutions using the effect of relaxation time of the heat flux. Literature shows more interest in developed identical studies in [5-7].

Ramped heating plays a good role in real-life problems such as diagnoses of prognosis, analysis of heart function, and blood vessel system [8-10]. Moreover, Kundu [11] investigates the thermal therapy based on ramped heating to destroy the cancer cells on the human structure. Initially, convective viscous fluid with ramped heating over vertical wall analyzes by Schertz [12] and Hayday [13]. The heat absorption ramped heating and thermal effect near a moving wall discussed by Seth et al. [14]. Further authors [15] investigate the dynamical aspect of mass and heat transformation with Darcy's law, chemical reaction, and ther-

E-mail: tauseefsaeed301@gmail.com

Muhammad Bilal Riaz, Department of Mathematics, University of Management and Technology, Lahore, 54000, Pakistan Institute for Groundwater Studies (IGS), University of the Free State, Bloemfontein, 9301, South Africa

*Corresponding Author: Dumitru Baleanu, Department of Mathematics, Cankaya University, Ankara, 06790, Turkey Institutes of Space Sciences, Magurele, Bucharest, 077125, Romania, E-mail: dumitru@cankaya.edu.tr 
mal conditions. Previously, there is less study which deals the parallel use of ramped heating with ramped velocity. It is complicated to apply these conditions, but they have broad significance as a physical aspect. Researchers investigated the ramped heating to investigate the flows of Newtonian and non-Newtonian [16-19]. In multiple subdivisions of emerging technologies, ramped wall velocity has found broad applications. For instance, in medical sciences, diagnoses of cardiovascular infections by means of treadmill testing (TT) or Ergo-meters is efficient employment of ramped velocity. Ramped velocity is a significant tool to recognize, determine medication, anticipate prognosis, and assess the working capability of blood vessels and heart.

The technique of fractional calculus has been used to formulate mathematical modeling in various technological development, engineering applications, and industrial sciences. Different valuable work has been discussed for modeling fluid dynamics, signal processing, viscoelasticity, electrochemistry, and biological structure through fractional time derivatives [20]. This fractional differential operator found useful conclusions for experts to treat cancer cells with a suitable amount of heat source and have compared the results to see the memory effect of temperature function. As compared to classical models, the memory effect is much stronger in fractional derivatives [21-25]. Over the last thirty years, Fractional derivative/calculus (FDs/FC) has captivated the numerous researchers after recognition of the fact that in comparison to the classical derivatives, FDs are more reliable operators to model the real-world physical phenomena. In dynamical problems, Fractional order models/ modeling are receiving a rapid popularity nowadays. The mathematical modeling of many physical and engineering models based on the idea of FC exhibits highly precise and accurate experimental results as compared to the models based on conventional calculus. For example, the fractional results of rate and differential type's fluids have a great resemblance with the results obtained experimentally. Tan [26], studied the generalized second grade fluid and learn the analytic solution of time dependent Couette flow. Riaz et al. [27] investigate the optimal solution of unsteady generalized second grade fluid via FD.

Riaz et al. [28] learn the view of newly FD operators of Maxwell fluid in heat and mass transfer study. They consider the Maxwell fluid and investigate the heat and mass transfer with the integer \& non-integer order derivative. Some further investigation of fluid flows and their properties equipped with FD establish in [29-45]. Applications of combined impact of heat and mass transfer in engineering, applied sciences and FC, since they are connected to the historical data (memory effect). Memory effect in FC means the occurrence of process depends not only in the present state but also on the past history of the process. FC has ability to remember prior effects of the input in order to calculate the current value of the output motivate us to investigate the time dependent natural convection flow of MHD Oldroyd-B fluid.

The intent of this manuscript is to explore the analytical solution of MHD OBM with simultaneous use of ramped heating with ramped velocity. New definitions of non-integer order derivatives $\mathrm{C}, \mathrm{CF}$ and $\mathrm{ABC}$ implemented using Laplace integral transformation is used to gain the solution of velocity, temperature and concentration under impact of simultaneous use of ramped conditions. In Section (2), the dimensionless governing equations are developed. In Sections (3), (4) and (5), non-integer order derivatives with Laplace integral transform is used to find the required solution of the concentration, temperature and velocity field. In Section (6), the effect of physical parameters is analyzed graphically. The concluding observation listed at the end.

\section{Problem statement}

We discussed the unsteady generalized OBF flow over an infinite plate. The flow representation and governing equations of $\mathrm{OBF}$ using geometry with appropriate conditions are analyzed in Figure 1 [33]:

$$
\begin{gathered}
\left(1+\lambda_{1} \frac{\partial}{\partial \tau}\right) \frac{\partial w(\eta, \tau)}{\partial \tau}=v\left(1+\lambda_{2} \frac{\partial}{\partial \tau}\right) \frac{\partial^{2} w(\eta, \tau)}{\partial \eta^{2}} \\
+g \beta\left(1+\lambda_{1} \frac{\partial}{\partial \tau}\right)\left(T-T_{\infty}\right)+g \beta\left(1+\lambda_{1} \frac{\partial}{\partial \tau}\right)\left(C-C_{\infty}\right) \\
-\left(1+\lambda_{1} \frac{\partial}{\partial \tau}\right) \frac{\sigma B_{0}^{2}}{\rho} w(\eta, \tau)-\left(1+\lambda_{2} \frac{\partial}{\partial \tau}\right) \frac{\mu \phi}{\rho k^{\star}} w(\eta, \tau) \\
\left(\rho C_{p}\right) \frac{\partial T(\eta, \tau)}{\partial \tau}=\kappa \frac{\partial^{2} T(\eta, \tau)}{\partial \eta^{2}} \\
\frac{\partial C(\eta, \tau)}{\partial \tau}=D_{m} \frac{\partial^{2} C(\eta, \tau)}{\partial \eta^{2}}
\end{gathered}
$$

$$
\begin{gathered}
\tau \leq 0, w(\eta, 0)=0, T(\eta, 0)=T_{\infty}, C(\eta, 0)=C_{\infty}, \frac{\partial w(\eta, 0)}{\partial \eta}= \\
\frac{\partial w(\eta, 0)}{\partial \tau}=0, \eta \geq 0, \tau>0, \\
w(0, \tau)=\left\{\begin{array}{l}
U_{0} \frac{\tau}{\tau_{0}} \text { for } 0<\tau \leq \tau_{o} \\
U_{0} \text { for } \tau>\tau_{o}
\end{array}\right. \\
T(0, \tau)=\left\{\begin{array}{l}
T_{\infty}+\left(T_{w}-T_{\infty}\right) \frac{\tau}{\tau_{0}} \text { for } 0<\tau \leq \tau_{o} ; \\
T_{w} \text { for } \tau>\tau_{o}
\end{array}\right.
\end{gathered}
$$




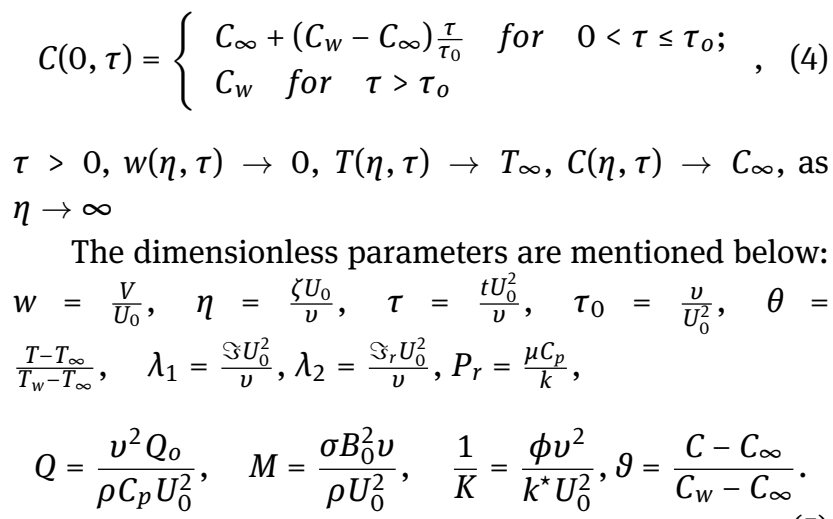

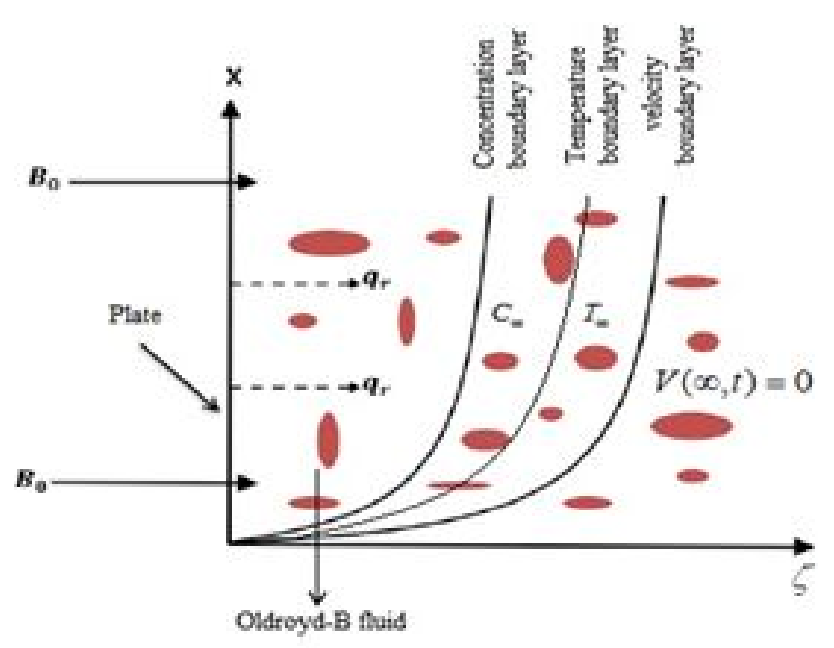

Figure 1: Geometry of Oldroyd-B model

Applying (5) into (1) - (4), we get the set of dimensionless governing equations with corresponding conditions,

$$
\begin{gathered}
\left(1+\Im \frac{\partial}{\partial t}\right) \frac{\partial V(\zeta, t)}{\partial t}=\left(1+\Im_{r} \frac{\partial}{\partial t}\right) \frac{\partial^{2} V(\zeta, t)}{\partial \zeta^{2}} \\
+G_{r}\left(1+\Im \frac{\partial}{\partial t}\right) \theta(\zeta, t)+G_{m}\left(1+\Im \frac{\partial}{\partial t}\right) \vartheta(\zeta, t) \\
-M\left(1+\Im \frac{\partial}{\partial t}\right) V(\zeta, t)-\frac{1}{K}\left(1+\Im_{r} \frac{\partial}{\partial t}\right) V(\zeta, t), \\
\frac{\partial \theta(\zeta, t)}{\partial t}=\frac{1}{P_{r}} \frac{\partial^{2} \theta(\zeta, t)}{\partial \zeta^{2}}, \\
\frac{\partial \vartheta(\zeta, t)}{\partial t}=\frac{1}{S_{c}} \frac{\partial^{2} \theta(\zeta, t)}{\partial \zeta^{2}}, \\
V(\zeta, 0)=\theta(\zeta, 0)=9(\zeta, 0)=0, \quad V_{t}(\zeta, 0)=V_{\zeta}(\zeta, 0)=0 \\
\text { for } \zeta \geq 0,
\end{gathered}
$$

$\vartheta(0, t)=\theta(0, t)=V(0, t)=\left\{\begin{array}{lll}t & \text { for } & 0<t \leq t_{o} ; \\ 1 & \text { for } t>t_{o}\end{array}\right.$,

$V(\zeta, t) \rightarrow 0, \quad \theta(\zeta, t) \rightarrow 0, \quad \vartheta(\zeta, t) \rightarrow 0, \quad$ for $\quad \zeta \rightarrow \infty$

\section{Solution of the temperature profile}

\subsection{Caputo time derivatives}

Fractional operators are quite flexible for describing the behaviors of heat transfer of MHD Oldroyd-B model through the characterization of governing equations of the temperature (7) via Caputo-fractional operator (11)

$$
{ }^{c} D_{\tau}^{\varepsilon} \theta(\zeta, \tau)=\frac{1}{P_{r}} \frac{\partial^{2} \theta(\zeta, \tau)}{\partial \zeta^{2}},
$$

where ${ }^{C} D_{\tau}^{\varepsilon}$ is called Caputo-Fractional operator [29] and its inverse defined below:

$$
\begin{aligned}
& { }^{C} D_{\tau}^{\varepsilon} M(\zeta, \tau)=\frac{1}{\Gamma(n-\varepsilon)} \int_{0}^{\tau}\left(\frac{M^{n}(t)}{(\tau-t)^{\varepsilon+1-n}}\right) d t, \\
& L\left\{{ }^{C} D_{\tau}^{\epsilon} M(\zeta, \tau)\right\}=\hbar^{\epsilon} L(M(\zeta, \tau))-h^{\epsilon-1} M(\zeta, 0) .
\end{aligned}
$$

Applying Eq. (12) to Eq. (10) with suitable condition on temperature, we have,

$$
\frac{\partial^{2} \hat{\theta}(\zeta, \hbar)}{\partial \zeta^{2}}-\left(P_{r} \hbar^{\varepsilon}\right) \widehat{\theta}(\zeta, \hbar)=0
$$

The required solution of Eq. (13) is written as:

$$
\bar{\theta}(\zeta, \hbar)=c_{1} e^{-\zeta \sqrt{P_{r} \hbar^{\epsilon}}}+c_{2} e^{\zeta \sqrt{P_{r} \hbar^{\epsilon}}} .
$$

We find the unknown using (9)

$$
\widehat{\theta}(\zeta, \hbar)=\left(\frac{1-e^{-\hbar}}{\hbar^{2}}\right) e^{-\zeta \sqrt{P_{r} \hbar^{\varepsilon}}}
$$

\subsection{Caputo-Fabrizio time derivatives}

Fractional operators are quite flexible for describing the behaviors of heat transfer of MHD Oldroyd-B model through the characterization of governing equations of temperature (7) via CF-fractional operator (17) of order $\varepsilon$.

$$
{ }^{C F} D_{\tau}^{\epsilon}(\zeta, \tau)=\frac{1}{P_{r}} \frac{\partial^{2} \theta(\zeta, \tau)}{\partial \zeta^{2}}
$$


where ${ }^{C F} D_{\tau}^{\varepsilon}$ is called Caputo-Fabrizio fractional operator [32] and its inverse defined below:

$$
\begin{gathered}
{ }^{C F} D_{\tau}^{\varepsilon} M(\zeta, \tau)=\frac{1}{1-\varepsilon} \int_{0}^{\tau} \exp \left(-\frac{\varepsilon(\tau-\varpi)}{1-\varepsilon}\right) M^{\prime}(\varpi) d \varpi, \\
L\left\{{ }^{C F} D_{\tau}^{\epsilon} M(\zeta, \tau)\right\}=\frac{\hbar L(M(\zeta, \tau))-M(\zeta, 0)}{(1-\epsilon) \hbar+\epsilon} .
\end{gathered}
$$

Applying Eq. (18) to Eq. (16) with suitable condition on temperature, we have,

$$
\frac{\partial^{2} \widehat{\theta}(\zeta, \hbar)}{\partial \zeta^{2}}-\left(P_{r} \frac{\hbar}{(1-\varepsilon) \hbar+\varepsilon}\right) \hat{\theta}(\zeta, \hbar)=0 .
$$

The required solution of Eq. (19) is written as:

$$
\widehat{\theta}(\zeta, \hbar)=c_{1} e^{-\zeta \sqrt{P_{r\left(\frac{\hbar}{(1-\varepsilon) \hbar+\varepsilon}\right.}}}+c_{2} e^{\zeta \sqrt{P_{r \frac{\hbar}{(1-\varepsilon) \hbar+\varepsilon}}}} .
$$

We find the unknown using (9)

$$
\widehat{\theta}(\zeta, \hbar)=\left(\frac{1-e^{-\hbar}}{\hbar^{2}}\right) e^{-\zeta \sqrt{P_{r \frac{\hbar}{(1-\varepsilon) \hbar+\varepsilon}}}}
$$

\subsection{Atangana-Baleanu time derivatives}

Fractional operators are quite flexible for describing the behaviors of heat transfer of MHD Oldroyd-B model through the characterization of governing equations of temperature (7) via $\mathrm{ABC}$-fractional operator (23) of order $\varepsilon$.

$$
{ }^{A B C} D_{\tau}^{\varepsilon} \theta(\zeta, \tau)=\frac{1}{P_{r}} \frac{\partial^{2} \theta(\zeta, \tau)}{\partial \zeta^{2}},
$$

where ${ }^{A B C} D_{\tau}^{\varepsilon}$ is called Atangana-Baleanu fractional operator $[33,34]$ and its inverse defined below:

$$
{ }^{A B C} D_{\tau}^{\varepsilon} M(\zeta, \tau)=\frac{N(\varepsilon)}{1-\varepsilon} \int_{0}^{\tau} E_{\varepsilon}\left(-\frac{\varepsilon(\tau-\varpi)^{\varepsilon}}{1-\varepsilon}\right) M^{\prime}(\varpi) d \varpi,
$$

$$
L\left\{{ }^{A B C} D_{\tau}^{\varepsilon} M(\zeta, \tau)\right\}=\frac{\hbar^{\varepsilon} L(M(\zeta, \tau))-\hbar^{\varepsilon-1} M(\zeta, 0)}{(1-\varepsilon) \hbar^{\varepsilon}+\varepsilon} .
$$

Applying Eq. (24) to Eq. (22) with suitable condition on temperature,

$$
\frac{\partial^{2} \widehat{\theta}(\zeta, \hbar)}{\partial \zeta^{2}}-\left(\operatorname{Pr} \frac{\hbar^{\varepsilon}}{(1-\varepsilon) \hbar^{\varepsilon}+\varepsilon}\right) \widehat{\theta}(\zeta, \hbar)=0 .
$$

The required solution of Eq. (25) is written as:

$$
\widehat{\theta}(\zeta, \hbar)=c_{1} e^{-\zeta \sqrt{\operatorname{Pr}_{r} \frac{\hbar^{\varepsilon}}{(1-\varepsilon) \hbar^{\varepsilon}+\varepsilon}}}+c_{2} e^{\zeta \sqrt{\operatorname{Pr}_{r} \frac{\hbar^{\varepsilon}}{(1-\varepsilon) \hbar^{\varepsilon}+\varepsilon}}} .
$$

We find the unknown using (9)

$$
\widehat{\theta}(\zeta, \hbar)=\left(\frac{1-e^{-\hbar}}{\hbar^{2}}\right) e^{-\zeta \sqrt{P_{r} \frac{\hbar^{\varepsilon}}{(1-\varepsilon) \hbar^{\varepsilon}+\varepsilon}}}
$$

\section{Solution of the concentration profile}

\subsection{Caputo time derivatives}

Fractional operators are quite flexible for describing the behaviors of heat transfer of MHD Oldroyd-B model through the characterization of governing equations of concentration (8) via Caputo-fractional operator,

$$
\frac{\partial^{2} \widehat{\vartheta}(\zeta, \hbar)}{\partial \zeta^{2}}-\left(S_{c} \hbar^{\varepsilon}\right) \widehat{\vartheta}(\zeta, \hbar)=0 .
$$

The required solution of Eq. (28) is written as:

$$
\widehat{\vartheta}(\zeta, \hbar)=c_{1} e^{-\zeta \sqrt{S_{c} \hbar^{\varepsilon}}}+c_{2} e^{\zeta \sqrt{S_{c} \hbar^{\varepsilon}}} .
$$

We find the unknown using (9)

$$
\widehat{\vartheta}(\zeta, \hbar)=\left(\frac{1-e^{-\hbar}}{\hbar^{2}}\right) e^{-\zeta \sqrt{S_{c} \hbar^{\varepsilon}}} .
$$

\subsection{Caputo-Fabrizio time derivatives}

Fractional operators are quite flexible for describing the behaviors of heat transfer of MHD Oldroyd-B model through the characterization of governing equations of concentration (8) via CF-fractional operator

$$
\frac{\partial^{2} \widehat{\vartheta}(\zeta, \hbar)}{\partial \zeta^{2}}-\left(S_{c} \frac{\hbar}{(1-\varepsilon) \hbar+\varepsilon}\right) \widehat{\vartheta}(\zeta, \hbar)=0 .
$$

The required solution of Eq. (31) is written as:

$$
\widehat{\vartheta}(\zeta, \hbar)=c_{1} e^{-\zeta \sqrt{S_{c \frac{\hbar}{(1-\varepsilon) \hbar+\varepsilon}}}}+c_{2} e^{\zeta \sqrt{S_{c\left(\frac{\hbar}{(1-\varepsilon) \hbar+\varepsilon}\right.}}} .
$$

We find the unknown using (9)

$$
\widehat{\vartheta}(\zeta, \hbar)=\left(\frac{1-e^{-\hbar}}{\hbar}\right) e^{-\zeta \sqrt{S_{\frac{c}{(1-\varepsilon) \hbar+\varepsilon}}}} .
$$

\subsection{Atangana-Baleanu time derivatives}

Fractional operators are quite flexible for describing the behaviors of heat transfer of MHD Oldroyd-B model through the characterization of governing equations of concentration (8) via $\mathrm{ABC}$-fractional operator

$$
\frac{\partial^{2} \widehat{\vartheta}(\zeta, \hbar)}{\partial \zeta^{2}}-\left(S_{c} \frac{\hbar^{\varepsilon}}{(1-\varepsilon) \hbar^{\varepsilon}+\varepsilon}\right) \hat{\vartheta}(\zeta, \hbar)=0 .
$$

The required solution of Eq. (34) is written as:

$$
\widehat{\vartheta}(\zeta, \hbar)=c_{1} e^{-\zeta \sqrt{S_{c \frac{\hbar^{\varepsilon}}{(1-\varepsilon) \hbar^{\varepsilon}+\varepsilon}}}}+c_{2} e^{\zeta \sqrt{S_{c \frac{\hbar^{\varepsilon}}{(1-\varepsilon) \hbar^{\varepsilon}+\varepsilon}}}} .
$$

We find the unknown using (9)

$$
\widehat{\vartheta}(\zeta, \hbar)=\left(\frac{1-e^{-\hbar}}{\hbar}\right) e^{-\zeta \sqrt{S_{c \frac{\hbar \varepsilon}{(1-\varepsilon) \hbar^{\varepsilon}+\varepsilon}}}} .
$$




\section{Solution of the velocity profile}

\subsection{Caputo time derivatives}

We apply Eq. (12) for the solutions of the Eq. (6),

$$
\begin{aligned}
& \left(1+\Im \hbar^{\varepsilon}\right)(\hbar+M) \widehat{V}(\zeta, \hbar)=\left(1+\Im_{r} \hbar^{\varepsilon}\right)\left(\frac{\partial^{2}}{\partial \zeta^{2}}-\frac{1}{K}\right) \widehat{V}(\zeta, \hbar) \\
& +G_{r}\left(1+\Im \hbar^{\varepsilon}\right) \widehat{\theta}(\zeta, \hbar)+G_{m}\left(1+\Im \hbar^{\varepsilon}\right) \widehat{\vartheta}(\zeta, \hbar) .
\end{aligned}
$$

The solution of homogeneous part of (37) is:

$$
\widehat{V}(\zeta, \hbar)=c_{1} e^{\zeta \sqrt{\left(\frac{\left(1+\Im \hbar^{\varepsilon}\right)(\hbar+M)}{1+\Im_{r} \hbar^{\sigma}}\right)+\frac{1}{K}}}+c_{2} e^{-\zeta \sqrt{\left(\frac{\left(1+\Im \hbar^{\varepsilon}\right)(\hbar+M)}{1+\Im_{r} \hbar^{\omega}}\right)+\frac{1}{K}}} .
$$

The general solution can be given as

$$
\begin{aligned}
& \widehat{V}(\zeta, \hbar)=c_{1} e^{\zeta \sqrt{\left(\frac{\left(1+\Im \hbar^{\varepsilon}\right)(\hbar+M)}{1+\Im_{r} \hbar^{\omega}}\right)+\frac{1}{K}}} \\
& +c_{2} e^{-\zeta \sqrt{\left(\frac{\left(1+\Im \hbar^{\varepsilon}\right)(\hbar+M)}{1+\Im_{r} \hbar^{\omega}}\right)+\frac{1}{K}}}-\Re_{1} e^{-\zeta \sqrt{P_{r} \hbar^{\varepsilon}}}-\Re_{2} e^{-\zeta \sqrt{S_{c} \hbar^{\varepsilon}}},
\end{aligned}
$$

$$
\begin{aligned}
& \widehat{V}(\zeta, \hbar)=\left(\frac{1-e^{-\hbar}}{\hbar^{2}}\right) e^{-\zeta \sqrt{\left(\frac{\left(1+\Im \hbar^{\varepsilon}\right)(\hbar+M)}{1+\Im_{r} \hbar^{\omega}}\right)+\frac{1}{K}}} \\
& +\Re_{1}\left\{e^{-\zeta \sqrt{\left(\frac{\left(1+\Im \hbar^{\varepsilon}\right)(\hbar+M)}{1+\Im_{r} \hbar^{\dagger}}\right)+\frac{1}{K}}}-e^{-\zeta \sqrt{P_{r} \hbar^{\varepsilon}}}\right\}
\end{aligned}
$$

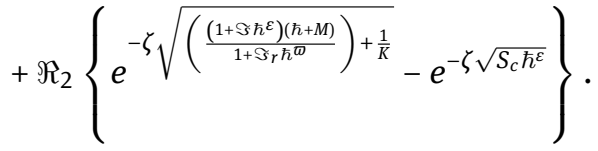

Where

$$
\begin{aligned}
& \Re_{1}=\frac{G_{r} K\left(1-e^{-\hbar}\right)\left(1+\Im_{r} \hbar^{\varpi}\right)\left(1+\Im \hbar^{\varepsilon}\right)}{\hbar^{2}\left[\left(P_{r} K \hbar^{\varepsilon}\left(1+\Im_{r} \hbar^{\varpi}\right)\right)-K\left(1+\Im^{\varepsilon}\right)(\hbar+M)-\left(1+\Im_{r} \hbar^{\varpi}\right)\right]}, \\
& \Re_{2}=\frac{G_{m} K\left(1-e^{-\hbar}\right)\left(1+\Im_{r} \hbar^{\varpi}\right)\left(1+\Im^{\varepsilon}\right)}{\hbar^{2}\left[\left(S_{c} K \hbar^{\varepsilon}\left(1+\Im_{r} \hbar^{\Phi}\right)\right)-K\left(1+\varsigma^{\varepsilon}\right)(\hbar+M)-\left(1+\Im_{r} \hbar^{\omega}\right)\right]} .
\end{aligned}
$$

\subsection{Caputo-Fabrizio time derivatives}

We apply Eq.(18) for the solutions of the Eq.(6),

$$
\begin{aligned}
& \left(1+\frac{\Im \hbar}{(1-\varepsilon) \hbar+\varepsilon}\right)(\hbar+M) \widehat{V}(\zeta, \hbar) \\
& =\left(1+\frac{\Im_{r} \hbar}{(1-\varpi) \hbar+\varpi}\right)\left(\frac{\partial^{2}}{\partial \zeta^{2}}-\frac{1}{K}\right) \widehat{V}(\zeta, \hbar) \\
& +G_{r}\left(1+\frac{\Im \hbar}{(1-\varepsilon) \hbar+\varepsilon}\right) \hat{\theta}(\zeta, \hbar) \\
& +G_{m}\left(1+\frac{\Im \hbar}{(1-\varepsilon) \hbar+\varepsilon}\right) \hat{\vartheta}(\zeta, \hbar) .
\end{aligned}
$$

The complementary solution of (41) is:

$$
\widehat{V}(\zeta, \hbar)=c_{1} e^{\zeta \sqrt{\left(\frac{\Re_{3}(\hbar+M)}{\Re_{4}}\right)+\frac{1}{K}}}+c_{2} e^{-\zeta \sqrt{\left(\frac{\Re_{3}(\hbar+M)}{\Re_{4}}\right)+\frac{1}{K}}} .
$$

The general solution can be given as

$$
\begin{aligned}
& \widehat{V}(\zeta, \hbar)=\left(\frac{1-e^{-\hbar}}{\hbar^{2}}\right) e^{-\zeta \sqrt{\left(\frac{\Re_{3}(\hbar+M)}{\Re_{4}}\right)+\frac{1}{K}}} \\
& +\Re_{5}\left\{e^{-\zeta \sqrt{\left(\frac{\Re_{3}(\hbar+M)}{\Re_{4}}\right)+\frac{1}{K}}}-e^{-\zeta \sqrt{P_{r} \frac{\hbar}{(1-\varepsilon) \hbar+\varepsilon}}}\right\} \\
& +\Re_{6}\left\{e^{-\zeta \sqrt{\left(\frac{\Re_{3}(\hbar+M)}{\Re_{4}}\right)+\frac{1}{K}}}-e^{-\zeta \sqrt{S_{c \frac{\hbar}{(1-\varepsilon) \hbar+\varepsilon}}}}\right\} \text {. }
\end{aligned}
$$

Where

$$
\begin{aligned}
\Re_{3}= & \frac{(1-\varepsilon+\Im) \hbar+\varepsilon}{(1-\varepsilon) \hbar+\varepsilon}, \Re_{4}=\frac{\left(1-\varpi+\Im_{r}\right) \hbar+\varpi}{(1-\varpi) \hbar+\varpi}, \\
& \Re_{5}=\frac{\Re_{3} \Re_{4} G_{r} K\left(1-e^{-\hbar}\right)((1-\varepsilon) \hbar+\varepsilon)}{\left.\hbar^{2}\left[\Re_{4} P_{r} K \hbar-K \Re_{3}(1-\varepsilon) \hbar+\varepsilon\right)(\hbar+M)-\Re_{4}((1-\varepsilon) \hbar+\varepsilon)\right]}, \\
& \Re_{6}=\frac{\Re_{3} \Re_{4} G_{m} K\left(1-e^{-}\right)((1-\varepsilon) \hbar+\varepsilon)}{\hbar^{2}\left[\Re_{4} S_{c} K \hbar-K \Re_{3}((1-\varepsilon) \hbar+\varepsilon)(\hbar+M)-\Re_{4}((1-\varepsilon) \hbar+\varepsilon)\right]} .
\end{aligned}
$$

\subsection{Atangana-Baleanu time derivatives}

We apply Eq.(18) for the solutions of the Eq.(6),

$$
\begin{aligned}
& \hat{V}(\zeta, \hbar)=\left(\frac{1-e^{-\hbar}}{\hbar^{2}}\right) e^{-\zeta \sqrt{\left(\frac{\Re_{7}(\hbar+M)}{\Re_{8}}\right)+\frac{1}{K}}}
\end{aligned}
$$

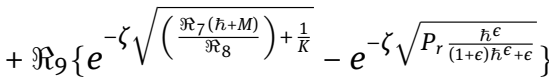

$$
\begin{aligned}
& +\Re_{10}\left\{e^{-\zeta \sqrt{\left(\frac{\Re_{7}(\hbar+M)}{\Re_{8}}\right)+\frac{1}{K}}}-e^{-\zeta \sqrt{S_{\epsilon} \frac{\hbar^{\epsilon}}{(1-\epsilon) \hbar^{\epsilon}+\epsilon}}}\right\}
\end{aligned}
$$

The complementary solution of (44) is:

$$
\widehat{V}(\zeta, \hbar)=c_{1} e^{\zeta \sqrt{\left(\frac{\Re_{7}(\hbar+M)}{\Re_{8}}\right)+\frac{1}{R}}}+c_{2} e^{-\zeta \sqrt{\left(\frac{\Re_{7}(\hbar+M)}{\Re_{8}}\right)+\frac{1}{R}}} .
$$

The general solution can be given as

$$
\begin{aligned}
& \widehat{V}(\zeta, \hbar)=\left(\frac{1-e^{-\hbar}}{\hbar^{2}}\right) e^{-\zeta \sqrt{\left(\frac{\Re_{7}(\hbar+M)}{\Re_{8}}\right)+\frac{1}{K}}}
\end{aligned}
$$

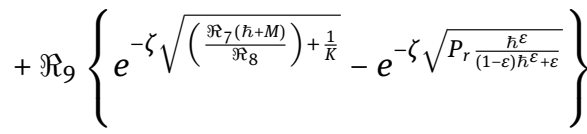

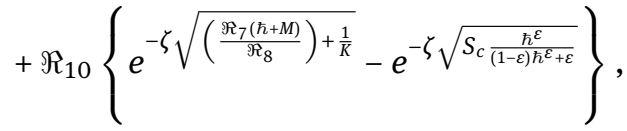

Where

$$
\begin{aligned}
& \Re_{7}=\frac{(1-\varepsilon+\Im) \hbar^{\varepsilon}+\varepsilon}{(1-\varepsilon) \hbar^{\varepsilon}+\varepsilon}, \\
& \Re_{8}=\frac{\left(1-\varpi+\Im_{r}\right) \hbar^{\varpi}+\varpi}{(1-\varpi) \hbar^{\varpi}+\varpi},
\end{aligned}
$$




$$
\begin{aligned}
& \Re_{9}=\frac{\Re_{7} \Re_{8} G_{r} K\left(1-e^{-\hbar}\right)\left((1-\varepsilon) \hbar^{\varepsilon}+\varepsilon\right)}{\hbar^{2}\left[\Re_{8} P_{r} K \hbar-K \Re_{7}\left((1-\varepsilon) \hbar^{\varepsilon}+\varepsilon\right)(\hbar+M)-\Re_{8}\left((1-\varepsilon) \hbar^{\varepsilon}+\varepsilon\right)\right]}, \\
& \Re_{10}=\frac{\Re_{7} \Re_{8} G_{m} K\left(1-e^{-\hbar}\right)\left((1-\varepsilon) \hbar^{\varepsilon}+\varepsilon\right)}{\hbar^{2}\left[\Re_{8} P_{r} K \hbar-K \Re_{7}\left((1-\varepsilon) \hbar^{\varepsilon}+\varepsilon\right)(\hbar+M)-\Re_{8}\left((1-\varepsilon) \hbar^{\varepsilon}+\varepsilon\right)\right]} .
\end{aligned}
$$

The numerical and computational techniques are used to evaluate the Laplace inverse via Stehfest's and Tzou's algorithms [46]. As $G_{m}=0$ and $(\varepsilon, \varpi) \rightarrow 1$, the required velocity equations (41), (45) and (49) we get the result discussed in [33]. Further if $\Im=0$ and $\Im_{r}=0$ then required results same as [16]. Moreover as $G_{m}=0$ then required results same as [19].

$$
\begin{aligned}
& v(\zeta, t)=\frac{\ln (2)}{t} \sum_{s=1}^{2 k} d_{s} \bar{v}\left(\zeta, s \frac{\ln (2)}{t}\right) \text {, with } \\
& d_{s}=(-1)^{s+k} \sum_{j=\left[\frac{s+1}{2}\right]}^{\min (s, k)} \frac{j^{n}(2 j) !}{(k-j) ! j !(j-1) !(s-1) !(2 j-s) !}
\end{aligned}
$$

\section{Validations of results}

a) If we neglect $G_{m}=0$ and $(\varepsilon, \varpi) \rightarrow 1$, then the required results are identical which obtained by [33].

b) If we neglect $G_{m}=0$, then the required results are identical which obtained by [19].

c) If we neglect $\Im=0$ and $\Im_{r}=0$, then required results are identical which obtained by [16].
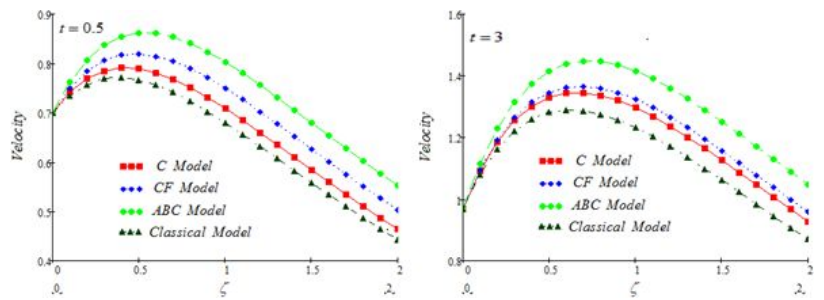

Figure 2: Comparison of velocity for integer \& non-integer derivative

\section{Results and discussion}

This part is devoted for physical interpretation of heat and mass transfer is executed on the motion of Oldroyd-B fluid near a porous surface. The impact of thermal radiation, magnetic field, and ramped conditions are also analyzed via Fractional derivative to obtain a solution via inversion algorithm. The impact of physical parameters such as $\operatorname{Pr}, M, G m, G r, K, \varepsilon, \varpi, \Im$ and $\Im r$ on energy, concentration and velocity profile are discussed using graphs. The time effect on all fractional derivative operators and classical model analyzed in figure (2). It is clearly show that
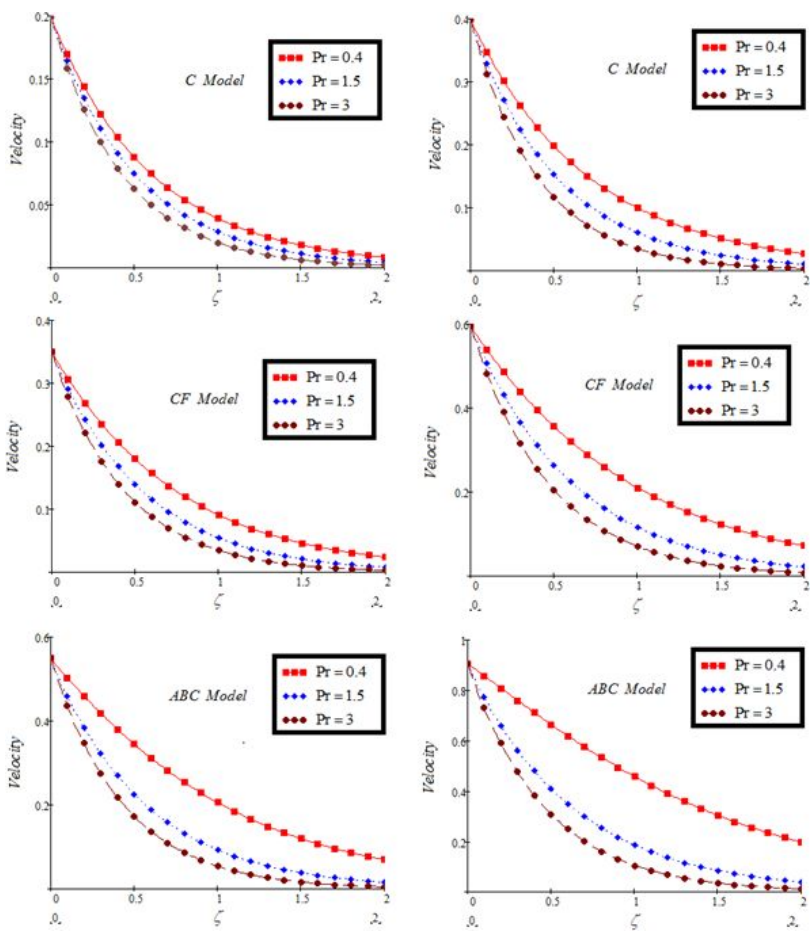

Figure 3: Velocity profile for FD's with variation of $\operatorname{Pr}$ and $\mathrm{Gr}=5$, $G m=2, \varepsilon=0.4$
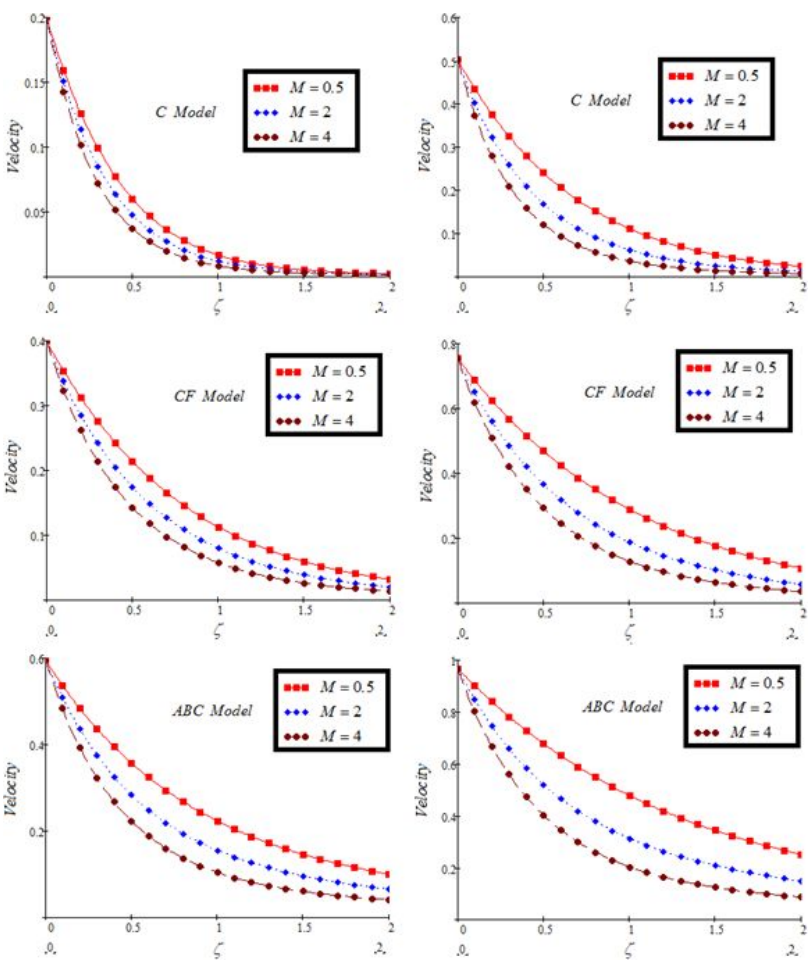

Figure 4: Velocity profile for FD's with variation of $M$ and $P r=0.7$, $G m=2, \varepsilon=0.5$

for altered time the behavior of velocity profile are same. The resultant velocity of $\mathrm{ABC}$ model is huge with respect 

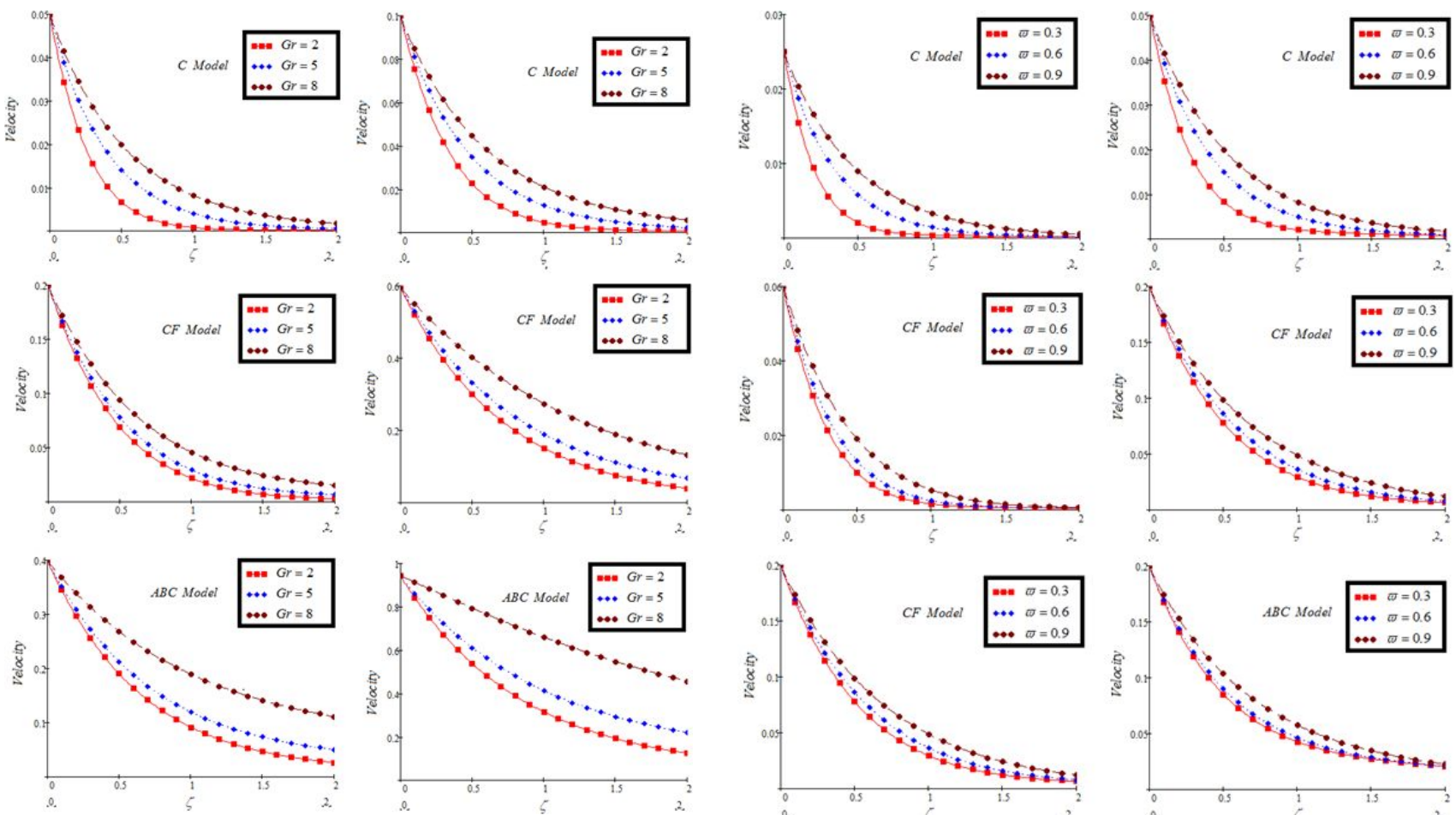

Figure 5: Velocity profile for FD's with variation of $G_{r}$ and $P r=0.7$, $M=2, \varepsilon=0.4$
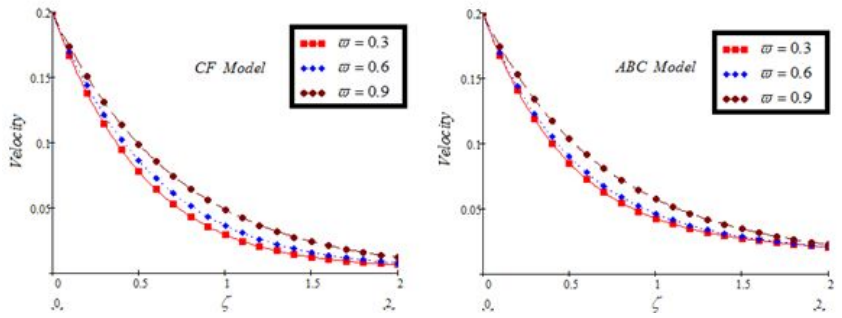

Figure 7: Velocity profile for FD's with variation of $\varpi$ and $\mathrm{Gr}=5$, $G m=2, \operatorname{Pr}=0.4$
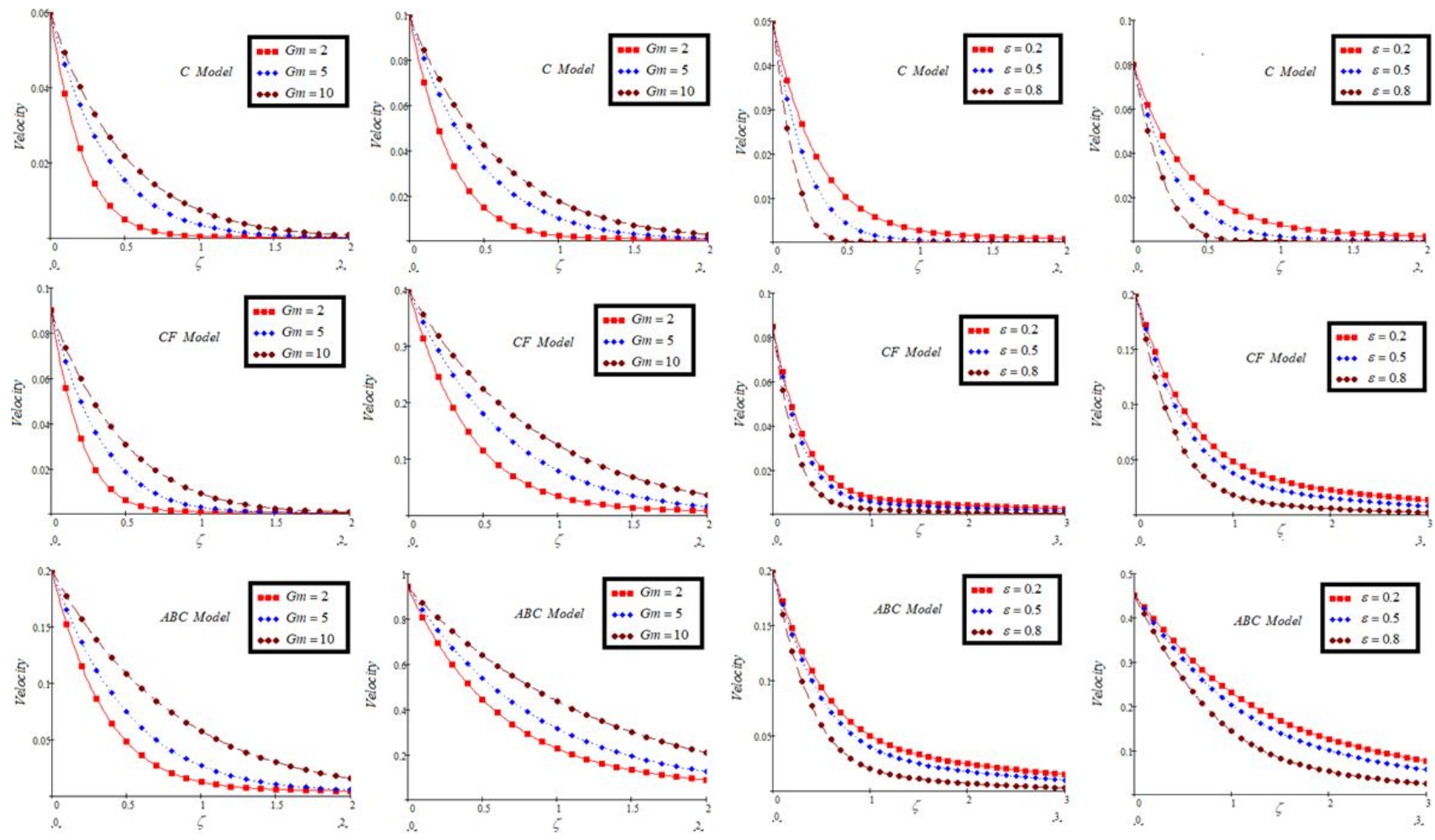

Figure 6: Velocity profile for FD's with variation of $G m$ and $P r=0.7$, $\Im=0.8, \varepsilon=0.6$
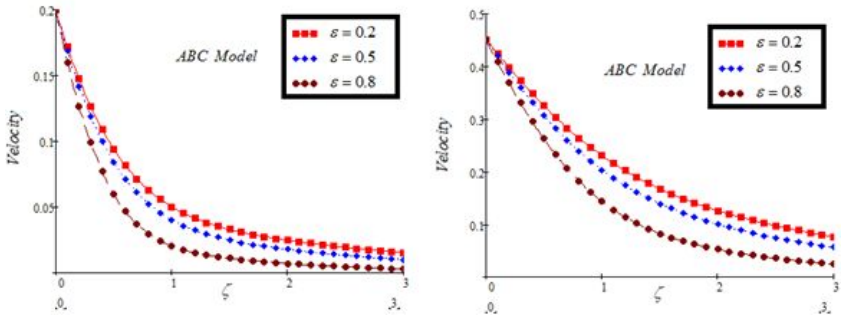

Figure 8: Velocity profile for FD's with variation of $\varepsilon$ and $G r=2$, $G m=8, \varepsilon=0.4$ 

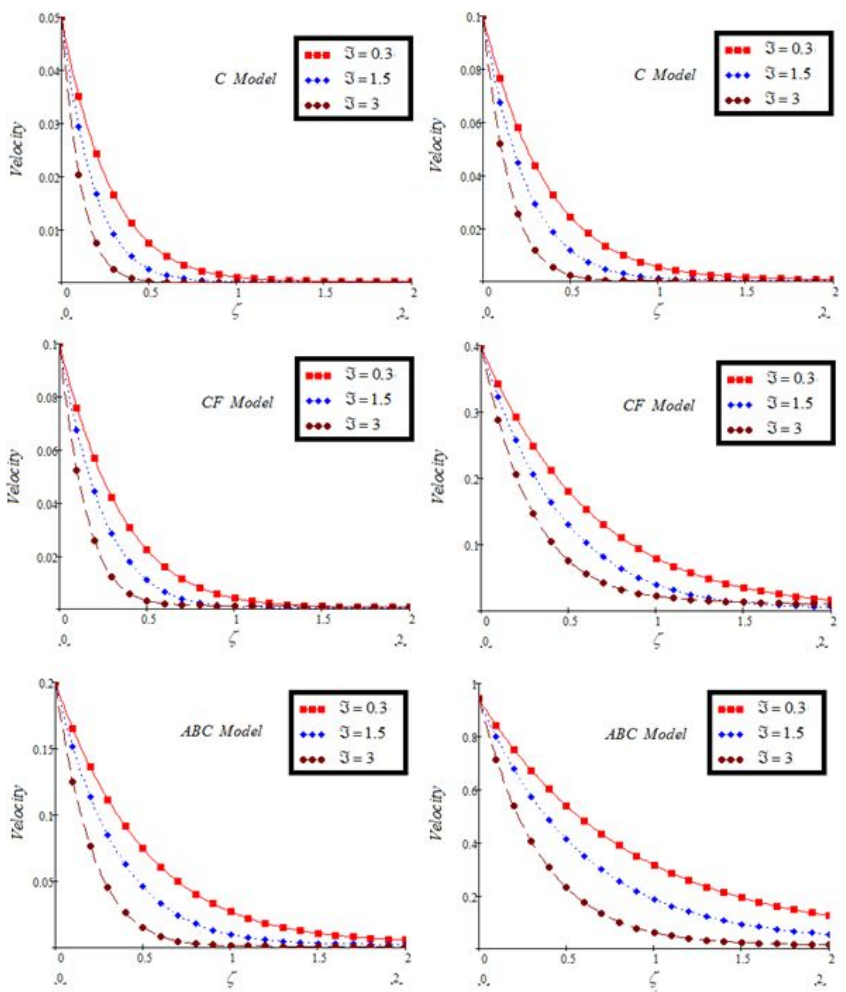

Figure 9: Velocity profile for FD's with variation of $\Im$ and $\operatorname{Pr}=2$, $M=0.5, \varepsilon=0.4$
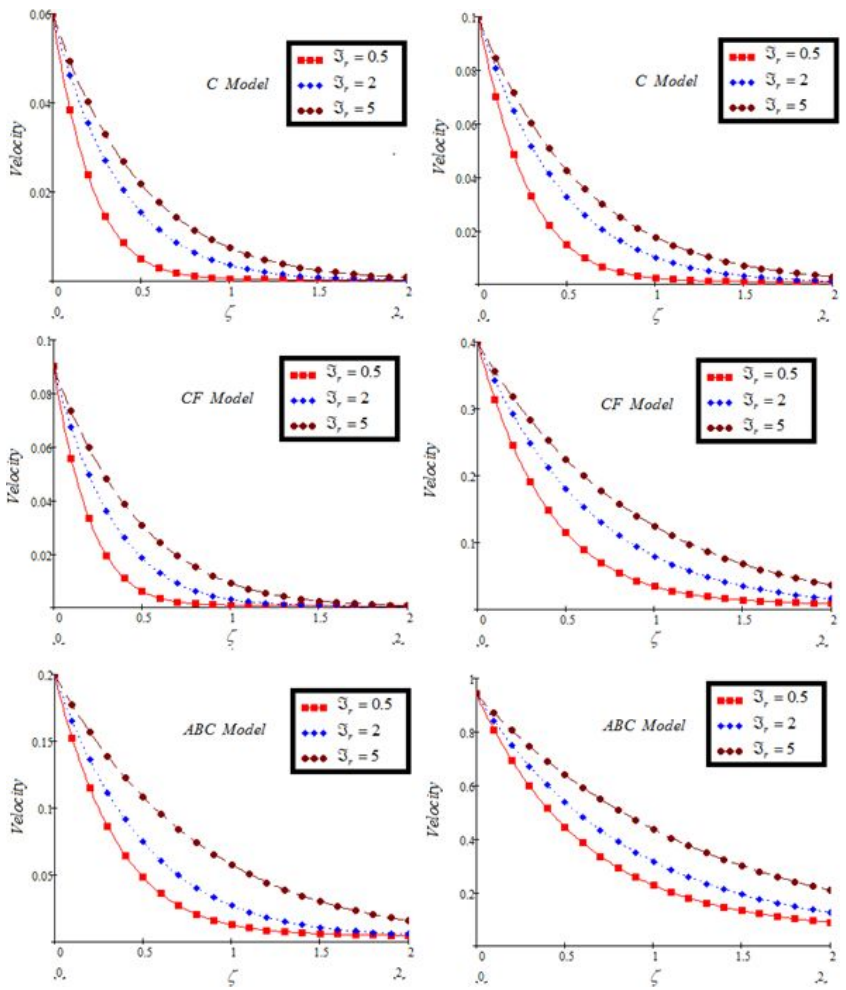

Figure 10: Velocity profile for FD's with variation of $\Im_{r}$ and $G r=5$, $G m=2, M=0.4$ to other fractional models as well as classical model. Figure (3) analyzes the behavior depends on Specific heat and conductivity of Pr. The velocity decreases as increase in the value of Pr. The lower Pr enhance the thermal conductivity and increase the boundary layer. Figure (4) investigate the domination of $\mathrm{M}$ on velocity components. The magnetic field increases as the velocity decreases. By enhancing the value of $\mathrm{M}$, the Lorentz force also increases. Due to this force the fluid flow on the boundary layer is slow down. It is perceive that the behavior of fluid profile for $\mathrm{ABC}$ model is effective as compared to other models. Thermal and isothermal conditions represent the domination of $\mathrm{Gr}$ shown in figures (5). Physically, $\mathrm{Gr}$ shows the relation between thermal forces to viscous force. For variation of time, the behavior of velocities is unique. The influence of $\mathrm{Gm}$ is illustrated in figure (6). It is notice that resultant velocity increase with enhance of in all fractional operators. It is also show that velocity increase with increase of time. The velocity field of ABC is huge as compared to Caputo and Caputo-Fabrizio. C, CF and ABC models analyzed the influence of fractional parameters $\varepsilon$ and $\vartheta$ on velocity via graphs as shown in figures (7) and (8). With large value of, the velocity profile also enhances. The behavior of velocity field is same for variation of time. Further memory effect of $\mathrm{ABC}$ is good as compared to other operators. Figure (8) represent the behavior of velocity profile for another fractional parameter $\varepsilon$. The behavior of $\varepsilon$ is reverse to $\vartheta$. With increase in $\varepsilon$, velocity field reduce for variation of time. Different physical properties are more effective to discuss in $\mathrm{ABC}$ model due to its non-local kernel. The influence of $\Im$ is illustrated in figure (9). It is notice that resultant velocity decrease with enhance of $\Im$ in all fractional operators due to thickness of boundary layer. It is also show that velocity increase with increase of time. Figures (10) show the effect of retardation time $\Im_{r}$. The behavior of $\Im$ and $\Im_{r}$ are reversible. Enhance $\Im_{r}$, the resultant velocity enhance with variation of time. The influence of all physical parameters on velocity profile using $A B C$ model is more effective as compared to other models.

\section{Conclusions}

The comprehensive analysis of the time fractional Analysis and heat transfer of a Oldroyd-B fluid in the presence of magnetic field with ramped conditions via has been investigated. To obtained the solution by using Laplace transformation, to compare the results between $\mathrm{C}, \mathrm{CF}$ and $\mathrm{ABC}$. To demonstrated in several graphs to analyze the effects of 
all parameters. The following major findings of this study are given below:

i) It is observed that the behaviors of fluid velocity for relaxation and retardation profiles are opposite to each other.

ii) Velocity behaves as a decreasing function for $\mathrm{M}$ and Pr.

iii) Increasing the worth of $\mathrm{Gr}$ and $\mathrm{Gm}$, the velocity profile also enhances.

iv) Velocity field for the $\mathrm{ABC}$ fractional operator is higher than the $\mathrm{CF}$ and Caputo fractional operator.

v) As the fractional parameter approaches to 1, then fractional models convert into classical model.

vi) Ramping of the enclosing wall is a salient technique to control the temperature and velocity of the fluid.

Acknowledgement: The authors are highly thankful and grateful to reviewers for support of this research article.

Funding information: The authors state no funding involved.

Author contributions: All authors have accepted responsibility for the entire content of this manuscript and approved its submission.

Conflicts of interest: The authors state no conflict of interest.

\section{References}

[1] Mousazadeh SM, Shahmardan MM, Tavangar T, Hosseinzadeh $\mathrm{K}$, Ganji DD. Numerical investigation on convective heat transfer over two heated wall-mounted cubes in tandem and staggered arrangement. Theo Appl Mech Lett. 2018;8(3):171-83.

[2] Sajad S, Nori A, Hosseinzadeh K, Ganji DD. Hydrothermal analysis of MHD squeezing mixture fluid suspended by hybrid nanoparticles between two parallel plates. Case Stud Thermal Eng. 2020;21:100650.

[3] Iftikhar N, Husnine SM, Riaz MB. Heat and mass transfer in MHD Maxwell fluid over an infinite vertical plate. J Prime Research Math. 2019;15:63-80.

[4] Qi H, Jin H. Unsteady rotating flows of a viscoelastic fluid with the fractional Maxwell model between coaxial cylinders. Acta Mech Sinica. 2006;22:301-5.

[5] Hosseinzadeh K, Mogharrebi AR, Asadi A, Paikar M, Ganji DD. Effect of fin and hybrid nano-particles on solid process in hexagonal triplex latent heat thermal energy storage system. J Mole Liquids. 2020;300:112347.

[6] Gholinia M, Gholinia S, Hosseinzadeh K, Ganji DD. Investigation on ethylene glycol nano fluid flow over a vertical permeable circular cylinder under effect of magnetic field. Res Phys.
2018;9:1525-33.

[7] Rahimi J, Ganji DD, Khaki M, Hosseinzadeh K. Solution of the boundary layer flow of an Eyring-Powell non-Newtonian fluid over a linear stretching sheet by collocation method. Alex Eng J. 2017;56:621-27.

[8] Sobral DC. A new proposal to guide velocity and inclination in the ramp protocol for the Treadmill Ergometer. Arq Bras de Card. 2003;81:48-3.

[9] Bruce RA. Evaluation of functional capacity and exercise tolerance of cardiac patients. Mod concept card disease. 1956;25:321-6.

[10] Myers J, Bellin D. Ramp exercise protocol for clinical and cardiopulmonary exercise testing. Sports Med. 2000;30:23-9.

[11] Kundu B. Exact analysis for propagation of heat in a biological tissue subject to different surface conditions for therapeutic applications. Appl Math Comp. 2016; 285:204-16

[12] Schetz JA. On the approximate solution of viscous flow problems. J Appl Mech. 1963;30:263-8.

[13] Hayday AA, Bowlus DA, McGraw RA. Free convection from a vertical flat plate with step discontinuities in surface temperature. J Appl Mech. 1967;89:244-9.

[14] Seth GS, Ansari MS. MHD natural convection flow past an impulsively moving vertical plate with ramped wall temperature in the presence of thermal diffusion with heat absorption. Int J Appl Mech Eng. 2010;15:199-15.

[15] Seth GS, Hussain SM, Sarkar S. Hydromagnetic natural convection flow with heat and mass transfer of a chemically reacting and heat absorbing fluid past an accelerated moving vertical plate with ramped temperature and ramped surface concentration through a porous medium. J Egy Math Soc. 2015;23:197-07.

[16] Seth GS, Nandkeolyar R, Ansari MS. Effect of rotation on unsteady hydro magnetic natural convection flow past an impulsively moving vertical plate with ramped temperature in a porous medium with thermal diffusion and heat absorption. Int J Appl Math Mech. 2011;7:52-69.

[17] Riaz MB, Atangana A, Saeed ST. MHD free convection flow over a vertical plate with ramped wall temperature and chemical reaction in view of non-singular kernel. In:Fractional Order Analysis: Theory, Methods and Applications. 2020;253-79. https://doi.org/10.1002/9781119654223.ch10.

[18] Anwar T, Khan I, Kumam P, Watthayu W. Impacts of Thermal Radiation and Heat Consumption/Generation on Unsteady MHD Convection Flow of an Oldroyd-B Fluid with Ramped Velocity and Temperature in a Generalized Darcy Medium. Mathematics. $2019 ; 8(1): 130$.

[19] Saeed ST, Khan I, Riaz MB, Husnine SM. Study of Heat Transfer under the Impact of Thermal Radiation, Ramped Velocity, and Ramped Temperature on the MHD Oldroyd-B Fluid Subject to non-integer Differentiable Operators. J Math. 2020;2020:1-14.

[20] Imran MA. Fractional Mechanism with Power Law (Singular) and Exponential (Non-singular) Kernels and Its Applications in Bio Heat Transfer Model. Int J Heat Tech. 2019;37 (3):846-52.

[21] Ahmad M, Imran MA, Aleem M, Khan I. A comparative study and analysis of natural convection flow of MHD non-Newtonian fluid in the presence of heat source and first-order chemical reaction. J Ther Anlay Cal. 2019;137(1):1783-96.

[22] Imran MA, Khan I, Ahmad M, Shah NA, Nazar M. Heat and mass transport of differential type fluid with non-intger order time fractional Caputo derivatives. J Mol Liquids. 
2017;229:67-5

[23] Imran MA, Shah NA, Aleem M, Khan I. Heat transfer analysis of fractional second grade fluid subject to Newtonian heating with Caputo and Caputo-Fabrizio fractional derivatives, A camparision. Eur Phy j Plus. 2017;132:340.

[24] Saeed ST, Abro KA, Almani S. Role of single slip assumption on the viscoelastic liquid subject to non-integer differentiable operators. Math Methods Appl Sci. 2021;44(7):6005-20.

[25] Iftikhar N, Saeed ST, Riaz MB. Fractional study of heat and mass transfer of MHD Oldroyd-B fluid with ramped velocity and temperature. Comp Methods Diff Equ. 2021;1-28. DOI:10.22034/cmde.2021.39703.1739.

[26] Tan W, Masuoka T. Stoke's first problem for a second grade fluid in a porous half-space with heated boundary. Int I nonLinear Mech. 2005;40(4):515-22.

[27] Riaz MB, Saeed ST, Baleanu D. Role of Magnetic field on the Dynamical Analysis of Second Grade Fluid: An Optimal Solution subject to Non-integer Differentiable Operators. J Appl Comp Mech. 2020;7(1):54-68.

[28] Riaz MB, Atangana A, Iftikhar N. Heat and mass transfer in Maxwell fluid in view of local and non-local differential operators. J Therm Anal Calorim. 2020;143:4313-29.

[29] Abdeljawad T, Riaz MB, Saeed ST, Iftikhar N. MHD Maxwell Fluid with Heat Transfer Analysis under Ramp Velocity and Ramp Temperature Subject to Non-Integer Differentiable Operators. Comp Model Eng Sci. 2021;126(2):821-41.

[30] Sene N. Second-grade fluid model with Caputo-Liouville generalized fractional derivative. Chao Solit Fract. 2020;133:109631.

[31] Fatecau C, Zafar AA, Vieru D, Awrejcewicz J. Hydromagnetic flow over a moving plate of second grade fluids with time fractional derivatives having non-singular kernel. Chao Solit Fract. 2020;133:109454.

[32] Siddique I, Tlili I, Bukhari M, Mahsud Y. Heat transfer analysis in convective flows of fractional second grade fluids with Caputo-Fabrizio and Atangana-Baleanu derivative subject to Newtonian heating. Mech Time-Depend Mater. 2019. https://doi.org/10.1007/s11043-019-09442-z.

[33] Tiwana MH, Mann AB, Rizwan M, Maqbool K, Javeed S, et al. Unsteady Magneto hydrodynamic Convective Fluid Flow of Oldroyd-B Model Considering Ramped Wall Temperature and Ramped Wall Velocity. Mathematics. 2019;7:676.

[34] Riaz MB, Saeed ST. Comprehensive analysis of integer order, Caputo-Fabrizio and Atangana-Baleanu fractional time derivative for MHD Oldroyd fluid with slip effect and time dependent boundary condition. Disc Cont Dyn Sys. 2020; https://doi.org/10.3934/dcdss.2020430.

[35] Saeed ST, Riaz MB, Baleanu D, Abro KA. A Mathematical Study of Natural Convection Flow through a Channel with nonsingular Kernels: An Application to Transport Phenomena. Alex Eng J. 2020;59(4):2269-81.

[36] Khan I, Saeed ST, Riaz MB, Abro KA, Husnine SM, et al. Influence in a Darcy's Medium with Heat Production and Radiation on MHD Convection Flow via Modern Fractional Approach. J Mater Res Tech. 2020;9(5):10016-30.

[37] Riaz MB, Siddiqui M, Saeed ST, Atangana A. MHD Oldroyd-B Fluid with Slip Condition in view of Local and Nonlocal Kernels. J Appl Comp Mech. 2020;7(1):116-27.

[38] Riaz MB, Saeed ST, Baleanu D, Ghalib M. Computational results with non-singular \& non-local kernel flow of viscous fluid in vertical permeable medium with variant temperature. Front Phys. 2020;8:275.

[39] Atangana A, Baleanu D. New fractional derivative with nonlocal and non-singular kernel: theory and application to heat transfer model. Therm Sci. 2016;20:763-9.

[40] Saeed ST, Riaz MB, Baleanu D, Akgul A, Husnine SM. Exact Analysis of Second Grade Fluid with Generalized Boundary Conditions. Intel Auto \& Soft Comp. 2021;28(2):547-59.

[41] Ahmed N, Shah NA, Vieru D. Natural convection with damped thermal flux in a vertical circular cylinder. Chin J Phys. 2018;56(2):630-44.

[42] Shah NA, Ahmed N, Vieru D, Fatecau C. Effects of double stratification and heat flux damping on convective flows over a vertical cylinder. Chin J Phys. 2019;60:290-06.

[43] Elazem Abd, Nader Y. Numerical results for influence the flow of MHD nanofluids on heat and mass transfer past a stretched surface. Nonlinear Eng. 2021;10(1):28-8.

[44] Jamil M, Haleem A. MHD fractionalized Jeffrey fluid over an accelerated slipping porous plate. Nonlinear Eng. 2020;9(1):27389.

[45] Ajibade AO, Umar AM. Mixed convection flow in a vertical channel in the presence of wall conduction, variable thermal conductivity and viscosity. Nonlinear Eng. 2020;9(1):412-31.

[46] Stehfest HA. Numerical inversions' of Laplace transforms. Comm ACM, 1970;13:9-47. 